\title{
Robust Curve Detection Using a Radon Transform in Orientation Space
}

\author{
M. van Ginkel ${ }^{1}$, M.A. Kraaijveld ${ }^{2}$, L.J. van Vliet $^{1}$, \\ E.P. Reding ${ }^{2}$, P.W. Verbeek ${ }^{1}$, and H.J. Lammers ${ }^{2}$ \\ 1 Pattern Recognition Group, Delft University of Technology, \\ Lorentzweg 1, 2628 CJ Delft, The Netherlands \\ \{michael, lucas\}@ph.tn.tudelft.nl \\ 2 Shell International E\&P, Technology Applications and Research, \\ Rijswijk, The Netherlands
}

\begin{abstract}
We present a novel approach to parameterised curve detection. The method is based on the generalised Radon transform, which is traditionally applied to a 2D edge/line map. The novelty of our method is the mapping of the original $2 \mathrm{D}$ image to a $3 \mathrm{D}$ orientation space, which then forms the input for the Radon transform. The orientation space representation can represent multiple intersecting structures and contains local orientation information. We demonstrate our approach on a problem in geology and show that we can detect curves in a heterogeneous and noisy background.
\end{abstract}

\section{Introduction}

The extraction of primitives from images is often a significant step in the analysis of an image. An import class of primitives is formed by parameterised curves. The most popular approach to the extraction of these curves from images appears under a number of different guises: template matching, probably the most descriptive denomination; the Radon transform based approach; and the Hough transform based approach. We discuss this approach in more detail in section 3 . In this paper we use the Radon transform nomenclature. Among the advantages of the Radon transform are its conceptual simplicity and robustness. Its main drawback is that it is a brute-force approach, requiring extensive computational resources. It has one further drawback: its applicability is restricted to line drawings or sketch-like images. The Radon transform is therefore usually preceded by an edge detector yielding the outlines of the objects. The central theme of this paper is the observation that, under some circumstances, important information may be lost in this first stage.

Lines and edges are the elements that convey most of the structural information in an image. It is therefore natural that edge detection has received so much attention in the image analysis literature. Edge detectors typically operate in a neighbourhood, so it is more accurate to talk about local line and edges. In many cases, a detector designed for a single line or edge within the analysis window suffices. However, as argued in [6], when a neighbourhood contains multiple lines and edges or when they occur against a complex background, a special approach 
is needed. Orientation space [6] is a convenient representation for dealing with such scenes. The orientation space idea dates back to [13], see also the references in $[6]$.

In section 2 we briefly review the orientation space concept. Next, we discuss how we use the Radon transform in conjunction with orientation space to benefit from the latter's advantages. We demonstrate our approach on geological data.

\section{Orientation space}

The three-dimensional orientation space representation $I^{[\phi]}(x, y, \phi)$ of a twodimensional image $I(x, y)$ records at each spatial position $(x, y)$ for each possible orientation $\phi \in[0 ; \pi)$ the amount of evidence for the presence of a structure, for example a line, with orientation $\phi$ at the location $(x, y)$. Succinctly put, orientation space consists of a full set of local orientation histograms. Figure 1a depicts the orientation space representation of a circle.

The example in figure 1 in section 3.2 is a mathematical abstraction with ideal mathematical curves and exact knowledge about the orientation. In practice, the orientation space representation is computed through convolution with an orientation selective filter $\Phi\left(x, y ; \phi, \sigma_{a}\right)$

$$
I^{[\phi]}(x, y, \phi)=I(x, y) * \Phi\left(x, y ; \phi, \sigma_{a}\right) .
$$

The orientation selective template filter $\Phi\left(x, y ; 0, \sigma_{a}\right)$ is rotated over an angle $\phi$ to obtain $\Phi\left(x, y ; \phi, \sigma_{a}\right)$.

Various choices and trade-offs are involved which are discussed in detail in [6]. Here we briefly mention the main points. The most important aspect is the orientation selectivity, which can be tuned through the parameter $\sigma_{a}$. There is a trade-off between the orientation selectivity and spatial localisation. To distinguish small orientation differences, a large analysis window is needed.

It is natural to separate the scale-dependent and the orientation-dependent properties of the filter. This achieved by choosing $\Phi\left(x, y ; \phi, \sigma_{a}\right)$ to be polar separable in the Fourier domain: $\mathcal{F}\{\Phi\}\left(f, \theta ; \phi, \sigma_{a}\right)=\Phi_{[f]}(f) \Phi_{[\theta]}\left(\theta-\phi ; \sigma_{a}\right)$.

In an implementation of these ideas we must work with a sampled version of $I^{[\phi]}(x, y, \phi)$. It is possible to guarantee that the continuous orientation space can be reconstructed from the sampled version by imposing constraints on the filter [6]. Essentially the filter must be steerable [4], an idea that dates back in a restricted form to [9].

A final aspect of the filter bank is that we use quadrature filters to deal simultaneously with lines and edges. Polar-separable quadrature filters were first introduced in [8].

\section{Detecting curves in complex backgrounds}

\subsection{Template matching, Radon and Hough}

The most natural way of looking at the Radon transform, when used to detect curves, is in terms of template matching. Let us consider templates $T(\boldsymbol{x} ; \boldsymbol{p})$ with 
a parameter vector $\boldsymbol{p}$. The matching criterion is the inner product between the templates $T$ and the image $I$. We compute the parameter response function (PRF) $P(\boldsymbol{p})$ on the parameter space which consists of all points $\boldsymbol{p}$. The PRF expresses the match between $T$ and $I$ for any given parameter vector $\boldsymbol{p}$ :

$$
P(\boldsymbol{p})=\int_{\mathbb{R}^{D}} T(\boldsymbol{x} ; \boldsymbol{p}) I(\boldsymbol{x}) d \boldsymbol{x} .
$$

with $D$ the dimensionality of the image. The $\operatorname{PRF} P(\boldsymbol{p})$ contains peaks at locations $\boldsymbol{p}$ for which the template fits the image data well: curve detection is reduced to the simpler problem of peak finding. Although this technique can be used to detect grey-value blobs in $I$, usually this technique is applied to an edge/line map $E(\boldsymbol{x})$ containing the outlines of the objects instead of directly to the image $I$ [11]. Let us now consider curve detection in this scheme. We represent the locus of the curve as $C(\boldsymbol{x} ; \boldsymbol{p})=0$. We obtain the templates $T(\boldsymbol{x} ; \boldsymbol{p})=\delta(C(\boldsymbol{x} ; \boldsymbol{p}))$. In case we are looking for straight lines in 2D, parameterised [11] using the distance to the origin $d$ and the angle of the line $\theta$, we have:

$$
\begin{aligned}
P(d, \theta) & =\int_{\mathbb{R}^{2}} \delta(x \cos \theta+y \sin \theta-d) E(x, y) d x d y \\
& =\int_{\mathbb{R}} E(d \cos \theta-s \sin \theta, d \sin \theta+s \cos \theta) d s=\mathcal{R}\{E\}(d, \theta),
\end{aligned}
$$

where $\mathcal{R}\{E\}$ denotes the Radon transform [10] of $E$ and $s$ the variable used to integrate along the projection lines. One may easily recognise the projective nature of the Radon transform in the second integral. For curves other than straight lines, the natural extension of the Radon transform, the generalised Radon transform, is to project along the given curves. This is consistent with the templace matching definition. The Radon transform and related transforms have been extensively studied in integral geometry [5].

Having established the link between template matching and the Radon transform, let us now examine how the Hough transform [7] fits in. The key is looking at (2) from a computational point of view. Essentially, $P(\boldsymbol{p})$ can be computed in two ways:

Reading paradigm: For each $\boldsymbol{p}$, collect all the values of $I(\boldsymbol{x})$, apply the template weights $T(\boldsymbol{x} ; \boldsymbol{p})$, and sum everything.

Writing paradigm: Initialise the entire $\operatorname{PRF} P(\boldsymbol{p})$ to zero. For each point $\boldsymbol{x}$ in the input image determine its contribution, weighted with $T(\boldsymbol{x} ; \boldsymbol{p})$, to each of the points in the PRF and update the PRF.

The Hough transform corresponds to the writing paradigm. The Hough/writing formalism obscures the simplicity of the underlying transformation and almost precludes a continuous formulation: compare the somewhat artificial definition in [1] to equations (2) and (3). It is therefore unfortunate that this formulation is pre-eminent in the literature, despite the fact that the equivalence of the Hough transform to template matching was reported 25 years ago [12,11], and the link between the Hough and Radon transforms soon after [3]. 


\subsection{A multi-orientation constrained Radon transform}

In this section we discuss the use of multi-orientation information for the detection of curves in complex backgrounds. In the Hough transform literature, uni-modal orientation information has been used extensively to constrain the Hough writing process in order to reduce clutter in parameter space, see for example [2]. In terms of the generalised Radon transform, the orientation constraint can be incorporated as follows:

$$
P(\boldsymbol{p})=\int_{(x, y) \text { on } c(\boldsymbol{p})} w(\phi(x, y), \gamma(x, y)) E(x, y) d x d y .
$$

The difference in orientation $\phi(x, y)$ of the image structure and the orientation $\gamma(x, y)$ of the curve weights the results through the function $w$.

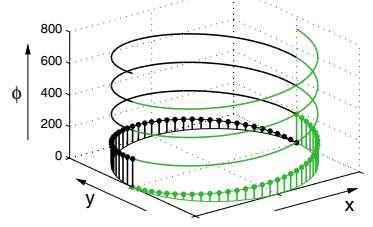

(a)

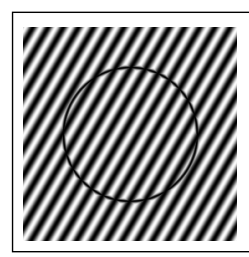

(b)

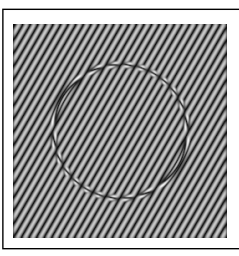

(c)

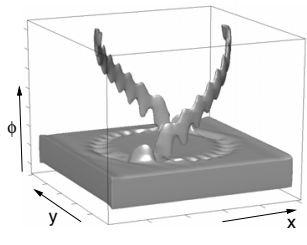

(d)

Fig. 1. a) Orientation space representation of a circle. Several periods of the curve along the $\phi$-axis are shown. The first period is displayed as a stem plot. Notice that the circle is transformed into a double helix. The units along the orientation axis are in degrees. b) Circle in a complex background. c) Standard (gradient magnitude) edge map of (b). d) Isosurface plot of the orientation space representation of (b). We can discern the double helix that corresponds to the circle in the input image, and a horizontal plane that represents the background pattern.

Uni-modal orientation information does not suffice for our purposes for two reasons. We want to extract multiple, intersecting, curves against a complex background. A schematic example is depicted in figure 1b. At intersections the result of a conventional orientation estimator will be unreliable: we may obtain some average orientation, or perhaps the estimator will "select" one of the orientations present. When the total contribution from intersecting points to the $\mathrm{PRF}$ is significant, we must have accurate orientation estimates at those points. Hence, a multi-orientation estimator is required. A second problem lies in the edge map. The Radon transform operates on the edge map. If the edge map cannot accurately represent the structures in the image, as illustrated in figure $1 \mathrm{~b}$ and $\mathrm{c}$, the whole approach will fail.

The key to resolving both problems is the orientation space representation. Instead of the standard edge map $E(x, y)$, we must use $I^{[\phi]}(x, y, \phi)$ as an improved edge map representation as depicted in figure $1 \mathrm{~d}$.

Obviously, the generalised Radon transforms for curves $\boldsymbol{c}(; \boldsymbol{p})$ cannot be directly applied to $I^{[\phi]}$. Instead, let us consider what happens to a curve $\boldsymbol{c}(s ; \boldsymbol{p})=$ 
$(x(s, \boldsymbol{p}), y(s, \boldsymbol{p}))$ under an orientation space mapping. For simplicity, we deal with ideal (zero-thickness), differentiable, curves and an ideal orientation space transformation. Each point $(x, y)$ is mapped to a new point $(x, y, \phi)$ in $I^{[\phi]}$ : it retains its spatial coordinates $(x, y)$ but a new coordinate $\phi$ is added. This coordinate is the angle of the tangent to the curve at $(x, y)$. If the curve is differentiable, $\phi$ can be computed from the parameterised description $\boldsymbol{c}(s ; \boldsymbol{p})$. In this way the entire curve $\boldsymbol{c}(s ; \boldsymbol{p})$ is mapped onto a new curve $\boldsymbol{c}^{[\phi]}(s ; \boldsymbol{p})=(x(s ; \boldsymbol{p}), y(s ; \boldsymbol{p}), \phi(s ; \boldsymbol{p}))$ in $I^{[\phi]}$.

The parameters of the original curves and those of the curves $\boldsymbol{c}^{[\phi]}$ are identical. Instead of detecting the curves $c$ directly in $I$, we can detect the curves $c^{[\phi]}$ in $I^{[\phi]}$ and obtain the same estimate for the parameters. This approach combines the strengths of the orientation space representation and those of the Radon transform. Summarising, our approach is comprised of two steps:

- Compute the orientation space $I^{[\phi]}(x, y, \phi)$. We use $I^{[\phi]}$ as edge map for use in the second stage. In this way we will use reliable orientation estimates for each individual curve even at intersection points and are capable of representing complex scenes that would be lost in conventional edge maps.

- Apply a generalised Radon transform for curves $\boldsymbol{c}^{[\phi]}(s ; \boldsymbol{p})$ to compute a PRF for the edge map $I^{[\phi]}(x, y, \phi)$ :

$$
P(\boldsymbol{p})=\int_{(x, y, \phi) \text { on } \boldsymbol{c}^{[\phi]}(; \boldsymbol{p})} I^{[\phi]}(x, y, \phi) d x d y d \phi .
$$

Each curve in the image yields a peak in the PRF, allowing us to detect the curves.

\section{Experiment}

We demonstrate our approach on an image analysis problem in geology: fracture detection in circumferential borehole images. These images are obtained by imaging the wall of an emptied borehole, which is modelled as a cylinder. Cylindrical coordinates $\psi$ (periodic) and $z$ (depth) are, therefore, a logical choice. Hence we denote these images as $B(\psi, z)$.

The subsurface is often made up of a stack of planes of different material, e.g. alternating layers of clay and sand. This regular structure is referred to as the bedding. Fractures are also planes, but where the bedding planes all share the same orientation, the fracture plane will usually have different orientation. Bedding planes and fracture planes intersecting the borehole will all show up as sinusoidal curves in the borehole image $B(\psi, z)$. Figure 2 shows the schematic structure of a borehole image and illustrates the necessity of the orientation space representation. In this space intersecting fractures and bedding planes lie apart. A parametric description $\boldsymbol{c}(\psi)$ of the sinusoidal curves is easily obtained:

$$
\boldsymbol{c}\left(\psi ; \psi_{d}, d, A\right)=(\psi, z(\psi)) \quad \text { with } \quad z(\psi)=A \sin \left(\psi-\psi_{d}\right)+d
$$

where we parameterise $\boldsymbol{c}$ as a function of the $\psi$ coordinate, rather than the distance along the curve. The parameters of the curve are the amplitude $A$, the 


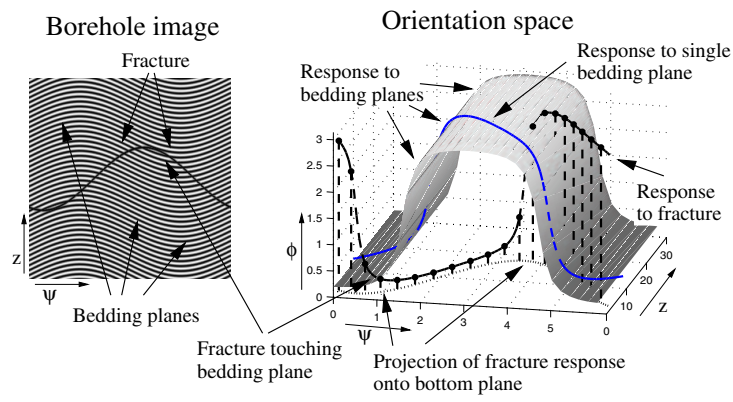

Fig. 2. Left: a schematic representation of a borehole image containing bedding and a single fracture. Right: the corresponding orientation space representation. Note that in orientation space the fracture intersects the bedding information at just two locations. The parameters of the sinusoids in the left and right pictures are not identical in order to improve the individual appearance of each of the plots.

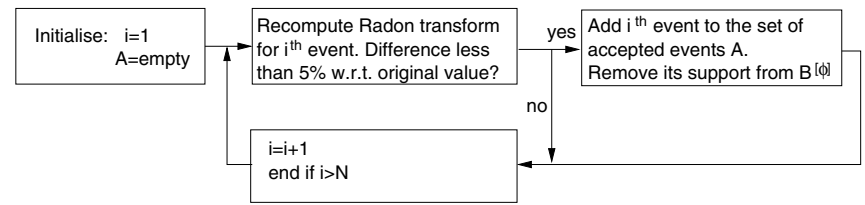

Fig. 3. The scheme to prevent multiple use of a single curve segment in the image as support for detection candidates. We have emperically established that the choice for the decision level of $5 \%$ is not a critical one.

phase $\psi_{d}$, and the depth $d$. The curve $\boldsymbol{c}$ is mapped onto the curve $\boldsymbol{c}^{[\phi]}$ in the orientation space $B^{[\phi]}(\psi, z, \phi)$ using the orientation space transformation:

$$
c^{[\phi]}\left(\psi ; \psi_{d}, d, A\right)=(\psi, z(\psi), \phi(\psi))=\left(\psi, z(\psi), \arg \left\{\left(-s_{\psi} \frac{d z(\psi)}{d \psi}, 1\right)\right\}\right) .
$$

The mismatch in units ( $z$ in pixels, $\psi$ in radians) is compensated through the factor $s_{\psi}$. Using this parameterisation we can compute the parameter space $P\left(\boldsymbol{p}=\psi_{d}, d, A\right)$ by applying the Radon transform to $B^{[\phi]}(\psi, z, \phi)$ :

$$
P\left(\boldsymbol{p}=\psi_{d}, d, A\right)=\int_{(\psi, z, \phi) \text { on } \boldsymbol{c}^{[\phi]}(\boldsymbol{p})} B^{[\phi]}(\psi, z, \phi) d \psi d z d \phi .
$$

The flanks of a sinusoid fit well for a wide range of parameter settings: the peaks in $P(\boldsymbol{p})$ can be quite extensive. Noise on the extended flanks of the peaks leads to false detections. To suppress these and select only the most salient curves, we use the procedure depicted in figure 3. The key step in this procedure is the removal from $B^{[\phi]}$ of the support for the accepted detections. In this way we impose that any given segment of support is used for the detection of only one curve. The number of detections $N$ is set before the procedure and is typically 100-200. The operator typically only looks at only the first 25-30 (the amount is controlled by the operator).

We applied our scheme to six real data sets and to a synthetic image with various levels of additive noise. The most interesting of these, a microresistivity 


\section{Borehole image Geologist's picks Algorithm's picks}

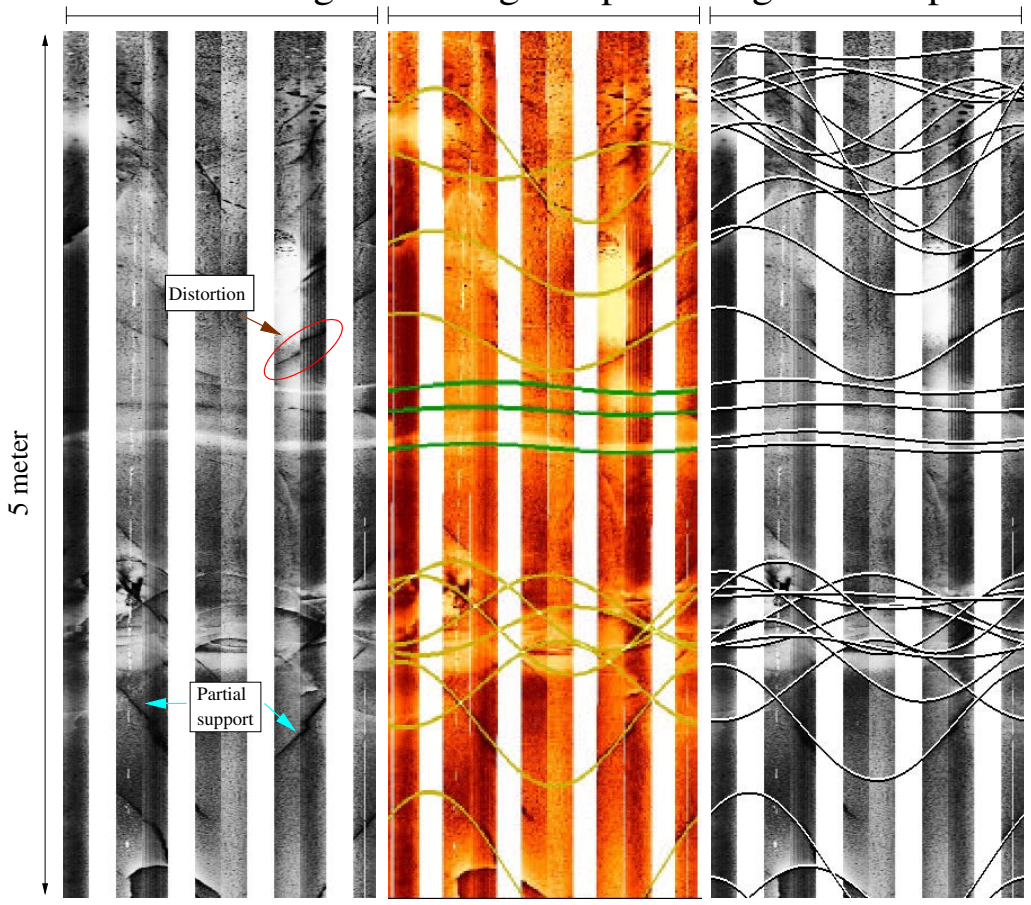

Fig. 4. Left: a $5 \mathrm{~m}$ interval of a micro resistivity image in a heavily fractured formation. Some typical problems with the data are indicated. Middle: the fractures identified by a geologist. Right: fractures detected by the algorithm.

image, is depicted in figure 4. This particular image contains very few bedding planes, but is interesting because of the large number of intersecting fractures. Large stripes of missing data can be observed: The imaging tool using eight pads which do not fully cover the borehole wall. The strong vertical edges are mapped onto known positions in orientation space and are easily ignored.

The image's dimensions are 292 by 2048 pixels. The orientation space was constructed using 17 ( $\sigma_{a} \approx 0.185 \mathrm{rad}$ ) oriented filters. The dimensions of $P\left(\psi_{d}, d\right.$, $A$ ) were $(73,512,50)$ pixels, with a sampling distance corresponding to four pixels in the original image. Our approach performs roughly as well as the geologist. The results for the synthetic data have shown that the accuracy is limited by the sampling distance of $P(\boldsymbol{p})$. Full experimental details and evaluation are in [6].

\section{Conclusions}

We have presented a new scheme for robust curve detection. We have argued that an alternative edge/line map is required for complex scenes: orientation space. Because the mapped curves have the same parameters as the curves in the original space, they can be extracted using a Radon transform applied to 
orientation space. In addition, we have shown an elegant geometric technique for adding the orientation constraint to the Radon transform.

The main drawback of the method is that it requires a large amount of computation. This becomes worse when we increase the dimensionality of the problem: an orientation space for a $D$-dimensional image has $2 D-1$ dimensions. The method also scales badly with respect to the complexity of the curve: the dimensionality of $P(\boldsymbol{p})$ is equal to the number of parameters of the curve.

With respect to the application of the scheme to the detection of fractures we conclude the detection performance is good and close to that of an experienced interpreter. Although an interpreter still has to verify the detected fractures, a significant reduction of the interpretation time is achieved. A patent is pending.

\section{Acknowledgements}

This work was partially supported by Shell International Exploration and Production B.V. and the Rolling Grants program 94RG12 of the Netherlands Organisation for Fundamental Research of Matter (FOM).

\section{References}

1. A.S. Aguado, E. Montiel, and M.S. Nixon. Bias error analysis of the generalised Hough transform. Journal of Mathematical Imaging and Vision, 12(1):25-42, 2000.

2. D.H. Ballard. Generalizing the Hough transform to detect arbitrary shapes. Pattern Recognition, 13(2):111-122, 1981.

3. S.R. Deans. Hough transform from the Radon transform. IEEE Transactions on Pattern Analysis and Machine Intelligence, 3(2):185-188, March 1981.

4. W.T. Freeman and E.H. Adelson. The design and use of steerable filters. IEEE Transactions on Pattern Analysis and Machine Intelligence, 13(9):891-906, 1991.

5. I.M. Gel'fand, M.I. Graev, and N.Ya. Vilenkin. Generalized Functions. Volume 5, Integral Geometry and Representation Theory. Academic Press, 1966.

6. M. van Ginkel. Image Analysis using Orientation Space based on Steerable Filters. PhD thesis, Delft University of Technology, Delft, The Netherlands, 2002. http://www.ph.tn.tudelft.nl/PHDTheses/MvGinkel/thesis_vanginkel.html.

7. P.V.C. Hough. Method and means for recognizing complex patterns. US patent nr. 3069654, 1962.

8. H. Knutsson and G. H. Granlund. Fourier domain design of line and edge detectors. In Proceedings of the 5th International Conference on Pattern Recognition, Miami, Florida, December 1980.

9. H. Knutsson, R. Wilson, and G.H. Granlund. Anisotropic non-stationary image estimation and its applications - Part I: Restoration of noisy images. IEEE Transactions on Communications, 31(3):388-397, March 1983.

10. J. Radon. Über die Bestimmung von Funktionen durch ihre Integralwerte längs gewisser Mannigfaltigkeiten. Berichte Sächsische Akademie der Wissenschaften, Leipzig, Mathematisch-Physikalische Klasse, 69:262-277, 1917.

11. J. Sklansky. On the Hough technique for curve detection. IEEE Transactions on Computers, 27(10):923-926, October 1978.

12. G.C. Stockman and A.K. Agrawala. Equivalence of Hough curve detection to template matching. Communications of the ACM, 20(11):820-822, 1977.

13. D. Walters. Selection of image primitives for general-purpose visual processing. Computer Vision, Graphics, and Image Processing, 37:261-298, 1987. 